\title{
Elastic Analysis for Nanocontact Problem with Surface Stress Effects under Shear Load
}

\author{
D. X. Lei, L. Y. Wang, and Z. Y. Ou \\ School of Science, Lanzhou University of Technology, Lanzhou 730050, China \\ Correspondence should be addressed to Z. Y. Ou, zhiyingou@163.com
}

Received 24 February 2012; Accepted 15 May 2012

Academic Editor: Sergio J. Mejía-Rosales

Copyright () 2012 D. X. Lei et al. This is an open access article distributed under the Creative Commons Attribution License, which permits unrestricted use, distribution, and reproduction in any medium, provided the original work is properly cited.

Consideration of surface stress effects on the elastic field of nanocontact problem has extensive applications in several modern problems of solid mechanics. In this paper, the effects of surface stress on the contact problem at nanometers are studied in the frame of surface elasticity theory. Fourier integral transform method is adopted to derive the fundamental solution of the nanocontact problem under shear load. As two special cases, the deformations induced by a uniformly distributed shear load and a concentrated shear force are discussed in detail, respectively. The results indicate some interesting characteristics in nanocontact mechanics, which are distinctly different from those in macrocontact problem. At nanoscale, both the contact stresses and the displacements on the deformed surface transit continuously across the uniform distributed shear load boundary as a result of surface stress. In addition, the indent depth and the contact stress depend strongly on the surface stress for nanoindentation.

\section{Introduction}

Contact problems of an elastic half-space by applied loads on its plane surface are the subject of extensive literature [1]. The greater parts of these works of literature are concerned with the responses subjected to surface loads with only existing residual surface tension [2]. Corresponding problems of complete effects of surface stress (nonzero surface tension and surface elastic properties) have also attracted the attention of a number of authors and a considerable body of literature exists on this class of problems [3]. The surface of solids is a special region with very small thickness (a few times of atom-spacing). Since the equilibrium lattice spacing in the surface is different from that in the bulk, surface stress appears. For solids with large characteristic dimensions, the volume ratios of surface region to the bulk material is small; the effect of surface stress then can be neglected because of its relatively tiny contribution. However, for microsolids with large surface-to-bulk ratio the significance of surfaces is likely to be important, such as nanocontact problem [4]. This is extremely true for nanoscale materials or structures.

When the characteristic size of an element or a solid approaches nanometers, its mechanical behavior displays remarkable size-dependent phenomena [5]. However, as there is no intrinsic length scale involved in the constitutive laws, the classical elastic theory cannot predict the sizedependent behavior of solids. Owing to the increasing ratio of surface-to-bulk volume, the effect of surface stress has been considered as one of the major factors contributing to the exceptional behaviors at nanoscale $[2,6-12]$.

To account for the effect of surface stress in solid mechanics, Gurtin and Ian Murdoch $[13,14]$ developed a continuum theory of surface elasticity. For some elementary deformation modes, the prediction of surface elasticity showed a good agreement with directly atomic simulation. Therefore, the surface elasticity has been widely adopted to investigate the mechanical phenomena at nanoscale. Miller and Shenoy [15] studied the size-dependent elastic stiffness of structural elements such as nanobars, nanobeams, and nanoplates. Cammarata et al. [16] considered the sizedependent deformation in thin film with surface effect. Through atomic simulation, Shenoy [17] calculated the surface elastic constants of metallic fcc crystal surfaces. Gao et al. [18] developed a finite-element method to account for the effect of surface elasticity. Zhang and Wang $[19,20]$ investigated the effect of surface energy on the yield strength of 
nanoporous materials and nanowires. Wang and Feng [2] studied the response of a half-plane subjected to normal pressures with constant residual surface tension. Ou et al. [911] studied how the size dependence can be considered in the mechanical performance of nanoscale structures and devices. Zhao and Rajapakse [21] derived the fundamental solution of an elastic layer bonded to a rigid substrate with surface effects. Due to the complexity of the problem, however, until now there has been no systematic investigation into the complete effects of stress (nonzero surface tension and surface elastic properties) on an isotropic elastic bulk. In this paper, Fourier integral transform method is used to solve the nonclassical boundary-value problems with complete surface effects.

In the present paper, the fundamental nanocontact problem of an isotropic elastic bulk with complete surface stress effects (nonzero surface tension and surface elastic properties) subjected to surface shear load is considered. The Fourier integral transform method is employed to obtain the fundamental solution of nanocontact problem with surface effects. This method allows us to easily extend our analysis to problems involving half-plane subjected to both concentrated shear load and uniformly distributed shear load on a finite region. The outline of the paper is organized as follows. The basic equations of surface elasticity are reviewed briefly in Section 2. In Section 3, the Fourier integral transform method is adopted to solve the nanocontact problem with surface effects subjected to shear loads. The detailed results for the cases of uniformly distributed shear load and concentrated forces are discussed in Sections 4 and 5, respectively, and concluding remarks are presented in Section 6.

\section{Basic Equations of Surface Elasticity}

In surface elasticity theory, a surface is regarded as a negligibly thin membrane that has material constants different from the bulk material and is adhered to the bulk without slipping. The equilibrium and constitutive equations in the bulk of material are the same as those in classical elastic theory, but the presence of surface stresses gives rise to a nonclassical boundary condition. For further details, the reader may refer to Gurtin and Ian Murdoch $[13,14]$. Here only several basic equations of surface elasticity theory are reviewed.

In the absence of body force, the equilibrium equations, constitutive law, and geometry relations in the bulk are as follows:

$$
\begin{gathered}
\sigma_{i j, j}=0 \\
\sigma_{i j}=2 G\left(\varepsilon_{i j}+\frac{v}{1-2 v} \varepsilon_{k k} \delta_{i j}\right),
\end{gathered}
$$

where $G$ and $v$ are the shear modulus and Poisson's ratio of the bulk material, $\sigma_{i j}$ and $\varepsilon_{i j}$ are the stress tensor and strain tensor in the bulk material, respectively. Throughout the paper, Einstein's summation convention is adopted for all repeated Latin indices $(1,2,3)$ and Greek indices $(1,2)$.

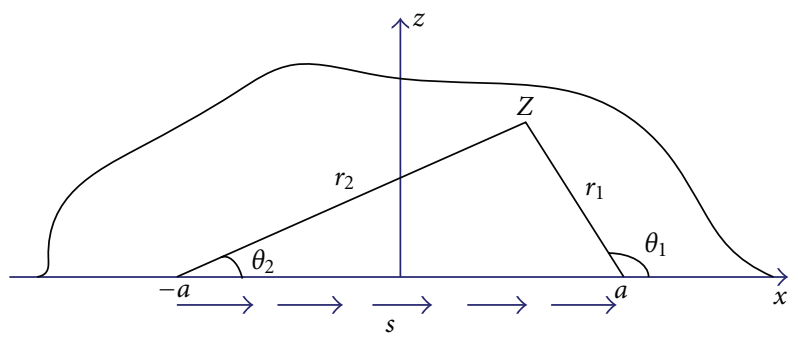

FigURE 1: Schematic of contact problem under uniform shear load. by

The strain tensor is related to the displacement vector $u_{i}$

$$
\varepsilon_{i j}=\frac{1}{2}\left(u_{i, j}+u_{j, i}\right)
$$

Assume that the surface of the material adheres perfectly to its bulk without slipping. Then the equilibrium conditions on the surface are expressed as

$$
\begin{gathered}
\sigma_{\beta \alpha} n_{\beta}+\sigma_{\beta \alpha, \beta}^{s}=0, \\
\sigma_{i j} n_{i} n_{j}=\sigma_{\alpha \beta}^{s} \kappa_{\alpha \beta},
\end{gathered}
$$

where $n_{i}$ denotes the normal to the surface, $\kappa_{\alpha \beta}$ the curvature tensor of the surface, and $\sigma_{\alpha \beta}^{s}$ the surface stress tensor.

The surface stress tensor is related to the surface strain tensor by

$$
\sigma_{\beta \alpha}^{s}=\tau^{s} \delta_{\beta \alpha}+2\left(\mu^{s}-\tau^{s}\right) \varepsilon_{\beta \alpha}+\left(\lambda^{s}+\tau^{s}\right) \varepsilon_{\gamma \gamma} \delta_{\beta \alpha},
$$

where $\tau^{s}$ is the residual surface tension under unstrained conditions, $\mu^{s}$ and $\lambda^{s}$ are surface Lamé constants which can be determined by atom simulations or experiments [17].

\section{Nanocontact Model with Surface Effects}

Considering a material occupying the upper half-plane $z>$ 0 , we refer to a Cartesian coordinate system $(o-x y z)$, as shown in Figure 1, where the $x$-axis is along the surface, and the $z$-axis perpendicular to the surface. The plane-strain conditions are assumed to $\varepsilon_{2 i}=0$, and the contact is assumed to be frictionless. In this case, the boundary conditions (3) on the contact surface $z=0$ are simplified to

$$
\begin{gathered}
\sigma_{z z}=0 \\
\sigma_{x z}+s(x)=-\left(\frac{d \tau^{s}}{d x}+k^{s} \frac{d^{2} u}{d x^{2}}\right),
\end{gathered}
$$

where $s(x)$ is the shear load applied on the surface, and $k^{s}=$ $2 \mu^{s}+\lambda^{s}$ is a surface material constant. 
For the considered plane problem, the equilibrium equations and Hooke's law in the bulk reduce to

$$
\begin{gathered}
\frac{\partial \sigma_{x x}}{\partial x}+\frac{\partial \sigma_{x z}}{\partial z}=0, \quad \frac{\partial \sigma_{z z}}{\partial z}+\frac{\partial \sigma_{x z}}{\partial x}=0, \\
\varepsilon_{x x}=\frac{1}{2 G}\left[(1-v) \sigma_{x x}-v \sigma_{z z}\right], \\
\varepsilon_{z z}=\frac{1}{2 G}\left[(1-v) \sigma_{z z}-v \sigma_{x x}\right], \\
\varepsilon_{x x}=\frac{\sigma_{x z}}{2 G} .
\end{gathered}
$$

The strains are related to the displacements by

$$
\varepsilon_{x x}=\frac{\partial u}{\partial x}, \quad \varepsilon_{z z}=\frac{\partial w}{\partial z}, \quad \varepsilon_{x z}=\frac{1}{2}\left(\frac{\partial u}{\partial z}+\frac{\partial w}{\partial x}\right) .
$$

which satisfy the following compatibility condition:

$$
\frac{\partial^{2} \varepsilon_{x x}}{\partial z^{2}}+\frac{\partial^{2} \varepsilon_{z z}}{\partial x^{2}}=2 \frac{\partial^{2} \varepsilon_{x z}}{\partial x \partial z} .
$$

As in classical theory of elasticity, the Airy stress function $\chi(x, z)$ is defined by

$$
\sigma_{x x}=\frac{\partial^{2} \chi}{\partial z^{2}}, \quad \sigma_{z z}=\frac{\partial^{2} \chi}{\partial x^{2}}, \quad \sigma_{x z}=-\frac{\partial^{2} \chi}{\partial x \partial z} .
$$

Then the equilibrium equations in (6) are satisfied automatically, and the compatibility equation in (9) becomes

$$
\left(\frac{\partial^{2}}{\partial x^{2}}+\frac{\partial^{2}}{\partial z^{2}}\right)\left(\frac{\partial^{2} \chi}{\partial x^{2}}+\frac{\partial^{2} \chi}{\partial z^{2}}\right)=0 .
$$

To solve the boundary-value problem, the Fourier integral transformation method is adopted to the coordinate $x$. Then, the Airy-stress function $\chi(x, z)$ and its Fourier transformation $\tilde{\chi}(\xi, z)$ can be expressed as

$$
\begin{aligned}
\tilde{\chi}(\xi, z) & =\frac{1}{\sqrt{2 \pi}} \int_{-\infty}^{\infty} \chi(x, z) e^{i x \xi} d \xi, \\
\chi(\xi, z) & =\frac{1}{\sqrt{2 \pi}} \int_{-\infty}^{\infty} \tilde{\chi}(x, z) e^{-i x \xi} d \xi .
\end{aligned}
$$

Substituting (12) into (11) and considering the condition that the stresses vanish at infinity, one obtains

$$
\tilde{\chi}(\xi, z)=(A+B z) e^{-z|\xi|},
$$

where $A$ and $B$ are generally functions of $\xi$ as yet to be determined.

Substituting (13) and (12) into (10), the stresses can be written as

$$
\begin{gathered}
\sigma_{x x}=\frac{1}{\sqrt{2 \pi}} \int_{-\infty}^{\infty} \xi^{2}[A(\xi)+(z-2|\xi|) B(\xi)] e^{-i x \xi-z|\xi|} d \xi, \\
\sigma_{z z}=-\frac{1}{\sqrt{2 \pi}} \int_{-\infty}^{\infty} \xi^{2}[A(\xi)+z B(\xi)] e^{-i x \xi-z|\xi|} d \xi, \\
\sigma_{x z}=\frac{i}{\sqrt{2 \pi}} \int_{-\infty}^{\infty} \xi[(1-|\xi| z) B(\xi)-|\xi| A(\xi)] e^{-i x \xi-z|\xi|} d \xi .
\end{gathered}
$$

By substituting the stresses into (7) and using (8), the displacements are derived as

$$
\begin{gathered}
\begin{aligned}
& w(x, z)=\frac{1}{2 \sqrt{2 \pi} G} \int_{-\infty}^{\infty}[|\xi| A(\xi)+(1-2 v+z|\xi|) B(\xi)] \\
& \times e^{-i x \xi-z|\xi|} d \xi, \\
& u(x, z)=\frac{i}{2 \sqrt{2 \pi} G} \int_{-\infty}^{\infty}[(2-v)|\xi| A(\xi)+(z|\xi|-2(1-v)) \\
&\times B(\xi)] e^{-i x \xi-z|\xi|} d \xi .
\end{aligned}
\end{gathered}
$$

Substituting (15) and (14) into the surface condition (5) leads to

$$
A(\xi)=0, \quad B(\xi)=i \frac{\tilde{s}(\xi)}{\xi} \frac{1}{1+b \xi} .
$$

Here

$$
\begin{gathered}
b=\frac{k^{s}(1-v)}{G}, \\
\widetilde{s}(\xi)=\frac{1}{\sqrt{2 \pi}} \int_{-\infty}^{\infty} s(x) e^{i x \xi} d x,
\end{gathered}
$$

where $b$ is a length parameter depending on the surface property and material elastic constants. It should be pointed out that this parameter indicates the thickness size of the zone where the surface effect is significant and plays a critical role in the surface elasticity. For metals, $b$ is estimated on the order of nanometers $[15,19,22]$.

In what follows, the two special cases including a uniform distributed shear load over a finite region and a concentrated shear force will be dicussed in detail, respectively.

\section{Uniform Distributed Shear Load}

In the case a uniform shear load $s$ acts over the region $|x| \leq a$, one has

$$
\widetilde{s}(\xi)=\sqrt{\frac{2}{\pi}} \frac{s \sin (a \xi)}{\xi} .
$$

Therefore $B(\xi)$ is given by

$$
B(\xi)=i \sqrt{\frac{2}{\pi}} \frac{s \sin (a \xi)}{\xi^{2}} \frac{1}{1+b \xi} .
$$

Then the stresses are obtained as

$$
\begin{gathered}
\sigma_{x x}=\frac{2 s}{\pi} \int_{0}^{\infty}\left[\frac{z \xi-2}{\xi(1+b \xi)}\right] \sin (a \xi) \sin (x \xi) e^{-z \xi} d \xi, \\
\sigma_{z z}=-\frac{2 s}{\pi} \int_{0}^{\infty}\left[\frac{z}{1+b \xi}\right] \sin (a \xi) \sin (x \xi) e^{-z \xi} d \xi, \\
\sigma_{x z}=-\frac{2 s}{\pi} \int_{0}^{\infty}\left[\frac{1-z \xi}{\xi(1+b \xi)}\right] \sin (a \xi) \sin (x \xi) e^{-z \xi} d \xi .
\end{gathered}
$$


The corresponding displacements are expressed as

$$
\begin{gathered}
u(x, z)=-\frac{s}{\pi G} \int_{0}^{\infty}\left[\frac{(2-v) z \xi-2(1-v)}{\xi^{2}(1+b \xi)}\right] \\
\times \sin (a \xi) \cos (x \xi) e^{-z \xi} d \xi \\
w(x, z)=\frac{s}{\pi G} \int_{0}^{\infty} \frac{1-2 v+z \xi}{\xi^{2}(1+b \xi)} \sin (a \xi) \sin (x \xi) e^{-z \xi} d \xi .
\end{gathered}
$$

On the contact surface $z=0$, the stresses is given by

$$
\begin{aligned}
& \sigma_{x x}=-\frac{4 s}{\pi} \int_{0}^{\infty}\left(\frac{b}{a} t+1\right)^{-1} \frac{\sin (t)}{t} \sin \left(\frac{x}{a} t\right) d t, \\
& \sigma_{x z}=-\frac{2 s}{\pi} \int_{0}^{\infty}\left(\frac{b}{a} t+1\right)^{-1} \frac{\sin (t)}{t} \cos \left(\frac{x}{a} t\right) d t .
\end{aligned}
$$

A closed-form solution for the elastic field of the halfplane cannot be obtained due to the complexity of the integrals involved in the solution. However, when $b=0$, that is, the surface influence is ignored in (22), the stresses of the half-plane are in agreement with those in the classical elastic results [1], and are expressed as

$$
\begin{aligned}
\sigma_{x x} & =\frac{s}{2 \pi}\left[4 \ln \left(\frac{r_{1}}{r_{2}}\right)-\left(\cos \left(2 \theta_{1}\right)-\cos \left(2 \theta_{2}\right)\right)\right], \\
\sigma_{x z} & =-\frac{s}{2 \pi}\left[2\left(\theta_{2}-\theta_{1}\right)+\left(\sin \left(2 \theta_{1}\right)-\sin \left(2 \theta_{2}\right)\right)\right],
\end{aligned}
$$

where $\tan \theta_{1,2}=z /(x \mp a)$ and $r_{1,2}=\left[(x \mp a)^{2}+z^{2}\right]^{1 / 2}$.

Assuming that the origin has no displacement in the $z$ direction, that is, $w(0,0)=0$, one obtains

$$
w(x, 0)=\frac{(1-2 v) a s}{\pi G} \int_{0}^{\infty}\left(\frac{b}{a} t+1\right)^{-1} \frac{\sin (t)}{t^{2}} \sin \left(\frac{x}{a} t\right) d t .
$$

If the shear displacement $u$ is specified to be zero at a distance $l$ on the contact surface, that is, $u(l, 0)=0$, the displacement on the surface is derived as

$$
\begin{aligned}
u(x, 0)=\frac{2(1-v) a s}{\pi G} \int_{0}^{\infty} & \left(\frac{b}{a} t+1\right)^{-1} \frac{\sin (t)}{t^{2}} \\
\times & {\left[\cos \left(\frac{x}{a} t\right)-\cos \left(\frac{l}{a} t\right)\right] d t . }
\end{aligned}
$$

It is instructive to examine the influence of the surface stress on the stresses and displacements of the contact surface and compare them with those in classical contact problem. Surface stress can be obtained from experiments [2] or atomic simulations [15]. According to these results, the influence of surface stresses, characterized by the intrinsic length, $b=k^{s}(1-v) / G$, becomes significant at nanoscale. For the illustration of the effects of surface energy, $b / a$ is taken as $0,0.2,0.5$, and 2 in our calculations. Figures 2 and 3 show the distribution of the stresses $\sigma_{x x}$ and $\sigma_{x z}$ on the contact surface, where the solution of $b / a=0$ is consistent with the classical elastic result. When the loading size $a$ is comparable to the parameter $b$, that is, of the order of nanometers, the influence

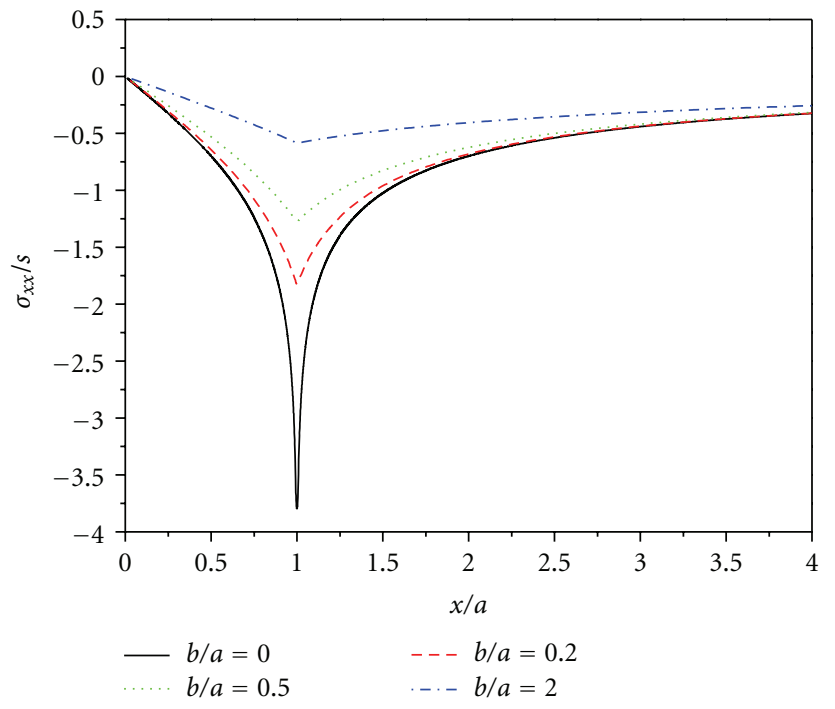

FIGURE 2: Distribution of contact normal stress under a uniform shear load.

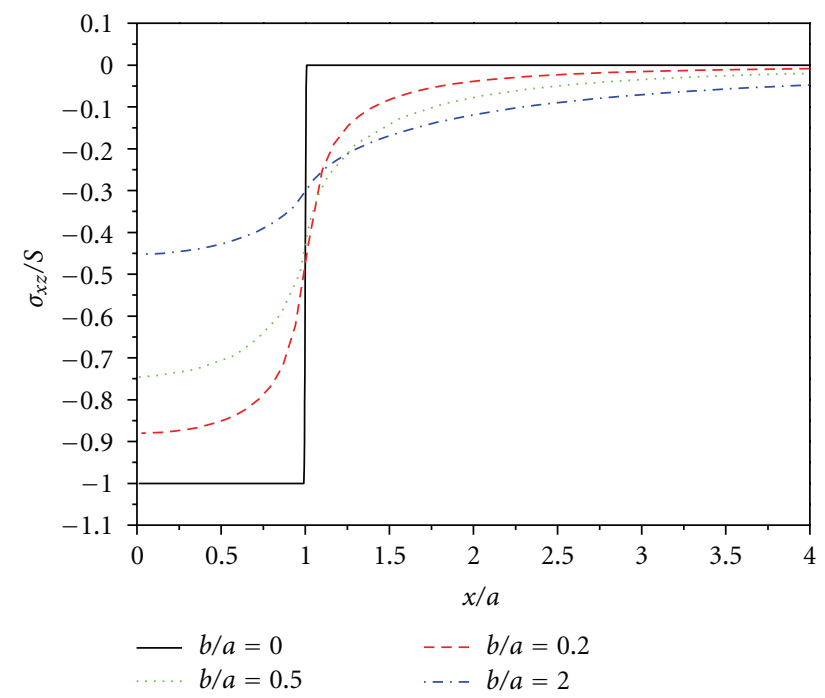

FIGURE 3: Distribution of contact shear stress under a uniform shear load.

of surface stress is evidently significant. It can be seen from Figure 2 that the normal stress transits continuously across the loading boundary $x= \pm a$, which is opposed to a singularity predicted by classical elasticity. In addition, the maximum normal stress in the bulk increases with an increase in surface stress. In the loading region $(|x / a|<1.0)$, the normal stress is decreasing monotonically with respect to $x$, and the inverse is observed outside the loading region $(|x / a|>1.0)$. It is also found in Figure 3 that shear stress changes smoothly across the loading boundary $x= \pm a$, which is different from a rapid jump from zero outside the load region to $s$ inside the load region predicted by the classical contact problem. In addition, the maximum shear 


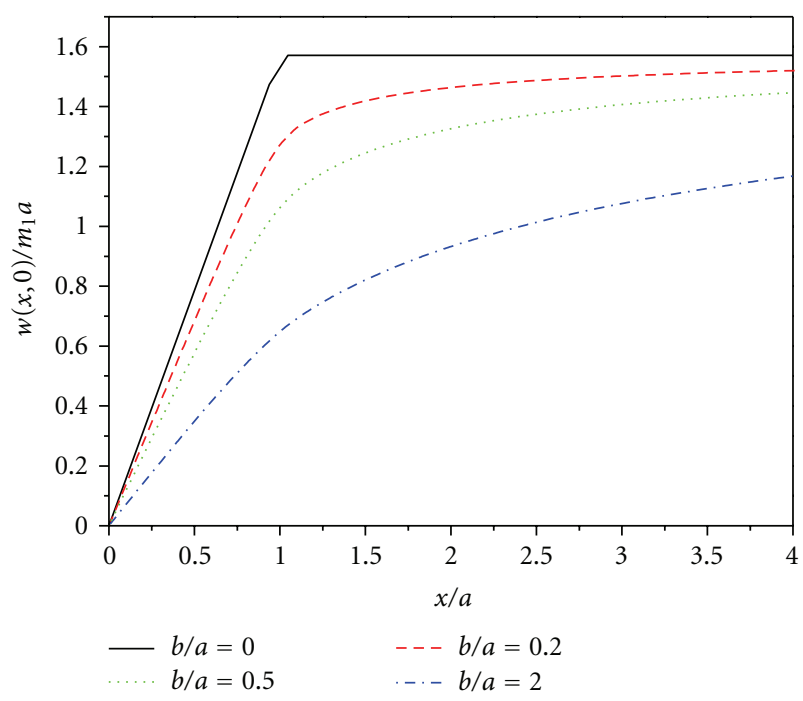

Figure 4: Surface displacement under a uniform shear load.

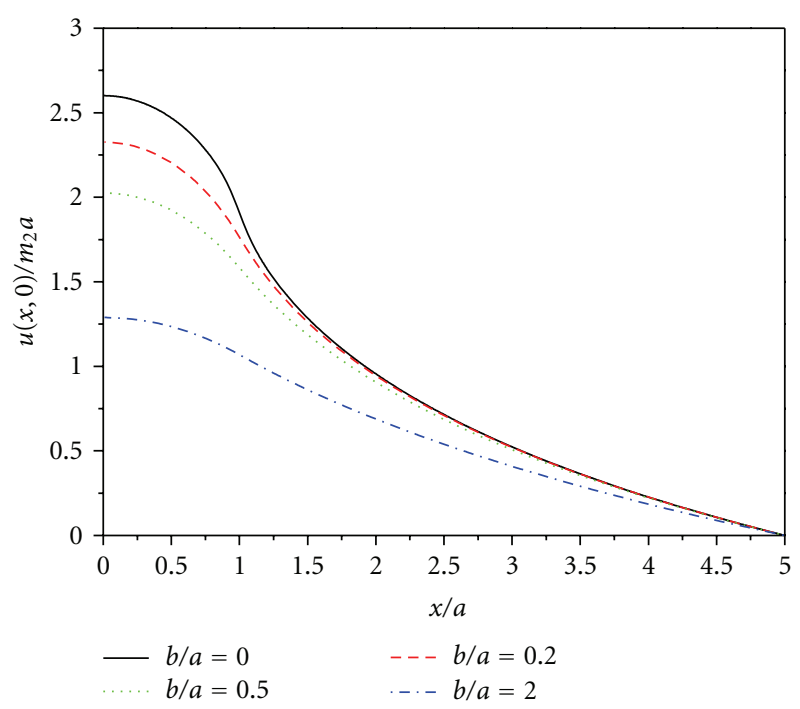

FIGURE 5: Surface indentation under a uniform shear load.

stress in the bulk decreases continuously with the increase of surface stress.

Due to the different surface stress value, the surface displacement on the contact surface is plotted in Figure 4 with $m_{1}=(1-2 v) s / \pi G$ which also shows that the gradient of the surface displacement is continuous everywhere on the deformed surface, though the classical elasticity gives only discontinuous displacement gradient at $x= \pm a$. With the increase of surface stress, the indent depth decreases continuously. The horizontal displacement is displayed in Figure 5, where we set $l=5 a$. and $m_{2}=2(1-v) s / \pi G$. It is seen that the slope of the deformed surface for $a>0$ is continuous everywhere. However, the classical elasticity theory predicted unreasonably that the gradient of the deformed surface is infinite at the load boundary $x= \pm a$, as seen from the curve of $b / a=0$.

\section{Concentrated Shear Load}

Now the extreme situation that a point force $S$ acts on the surface at the origin is considered by setting $2 s a \rightarrow S$ as $a \rightarrow$ 0 . Thus, one has

$$
B(\xi)=i \sqrt{\frac{1}{2 \pi}} \frac{S}{\xi} \frac{1}{1+b \xi} .
$$

Substituting (26) into (14) and (15), the corresponding stress and displacement distributions are given as follows, respectively,

$$
\begin{gathered}
\sigma_{x x}=\frac{S}{\pi} \int_{0}^{\infty} \frac{z \xi-2}{1+b \xi} \sin (x \xi) e^{-z \xi} d \xi, \\
\sigma_{z z}=-\frac{S}{\pi} \int_{0}^{\infty} \frac{z \xi}{1+b \xi} \sin (x \xi) e^{-z \xi} d \xi, \\
\sigma_{x z}=-\frac{S}{\pi} \int_{0}^{\infty} \frac{1-z \xi}{1+b \xi} \cos (x \xi) e^{-z \xi} d \xi, \\
u(x, z)=-\frac{S}{\pi G} \int_{0}^{\infty} \frac{(2-v) z \xi-2(1-v)}{\xi(1+b \xi)} \cos (x \xi) e^{-z \xi} d \xi, \\
w(x, z)=\frac{S}{\pi G} \int_{0}^{\infty} \frac{1-2 v+z \xi}{\xi(1+b \xi)} \sin (x \xi) e^{-z \xi} d \xi .
\end{gathered}
$$

However, when $b=0$, which implies that the surface influence is ignored, the stresses of the half-plane can be obtained as

$$
\begin{aligned}
\sigma_{x x} & =-\frac{2 S}{\pi} \frac{x^{3}}{\left(x^{2}+z^{2}\right)^{2}}, \\
\sigma_{x z} & =-\frac{2 S}{\pi} \frac{x^{2} z}{\left(x^{2}+z^{2}\right)^{2}}
\end{aligned}
$$

which are consistent with those in classical contact mechanics [1].

On the contact surface, the stresses and displacements are

$$
\begin{gathered}
\sigma_{x x}=-\frac{G}{1-v} \int_{0}^{\infty}\left(\frac{b}{\Lambda_{2}} t+1\right)^{-1} \sin \left(\frac{x}{\Lambda_{2}} t\right) d t, \\
\sigma_{x z}=-\frac{G}{1-2 v} \int_{0}^{\infty}\left(\frac{b}{\Lambda_{2}} t+1\right)^{-1} \cos \left(\frac{x}{\Lambda_{2}} t\right) d t, \\
u(x, 0)=\Lambda_{1} \int_{0}^{\infty} \frac{1}{t}\left(\frac{b}{\Lambda_{2}} t+1\right)^{-1}\left[\cos \left(\frac{x}{\Lambda_{2}} t\right)-\cos \left(\frac{l}{\Lambda_{2}} t\right)\right] d t, \\
w(x, 0)=\Lambda_{2} \int_{0}^{\infty} \frac{1}{t}\left(\frac{b}{\Lambda_{2}} t+1\right)^{-1} \sin \left(\frac{x}{\Lambda_{2}} t\right) d t,
\end{gathered}
$$

where

$$
\Lambda_{1}=\frac{2(1-v) S}{\pi G}, \quad \Lambda_{2}=\frac{(1-2 v) S}{\pi G} .
$$

The variation of the distribution of stresses on the contact surface subjected to a concentrated shear force with 


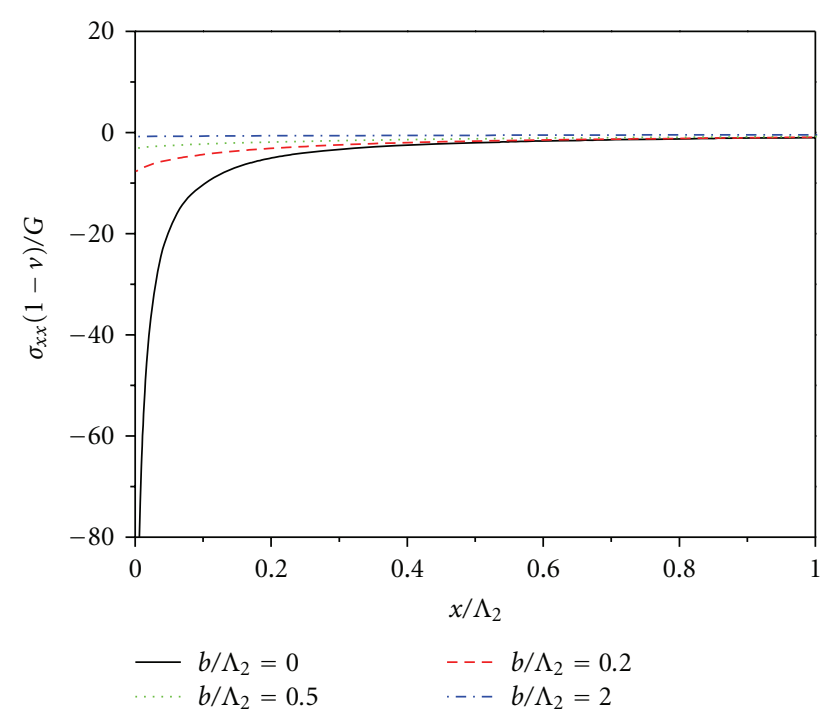

FIGURE 6: Distribution of the contact normal stress under a concentrated shear load.

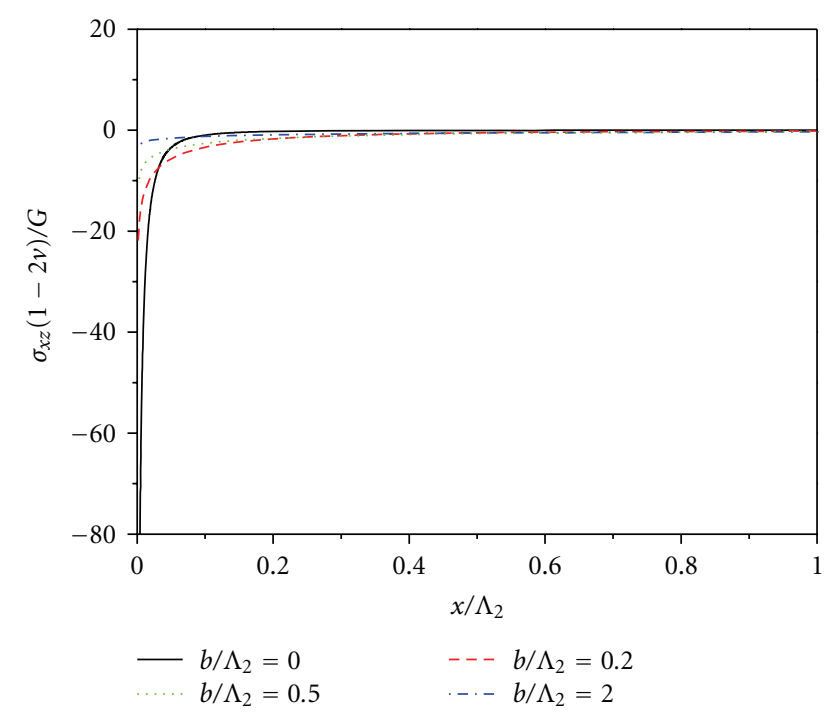

Figure 7: Distribution of the contact shear stress under a concentrated shear load.

various values of $b / \Lambda_{2}$ is shown in Figures 6 and 7. In classical elasticity without surface effect (i.e., $b / \Lambda_{2}=0$ ), the stresses tend to infinity at the loading point $x=0$, while it approaches finite values when surface stresses are present. And the stresses $\sigma_{x z}$ and $\sigma_{x x}$ at $x=0$ decrease with the increase of surface stress characterized by $b / \Lambda_{2}$. Figure 8 shows the maximum surface displacement in the bulk decreases with the increase of surface stress. Figure 9 shows the surface indentation for several representative values of surface stresses. Different from the classical elastic solution without surface effects (i.e., $b / \Lambda_{2}=0$ ), in which the indent depth at $x=0$ is maximum, the indent depth will decreases when the effect of surface stress is taken into

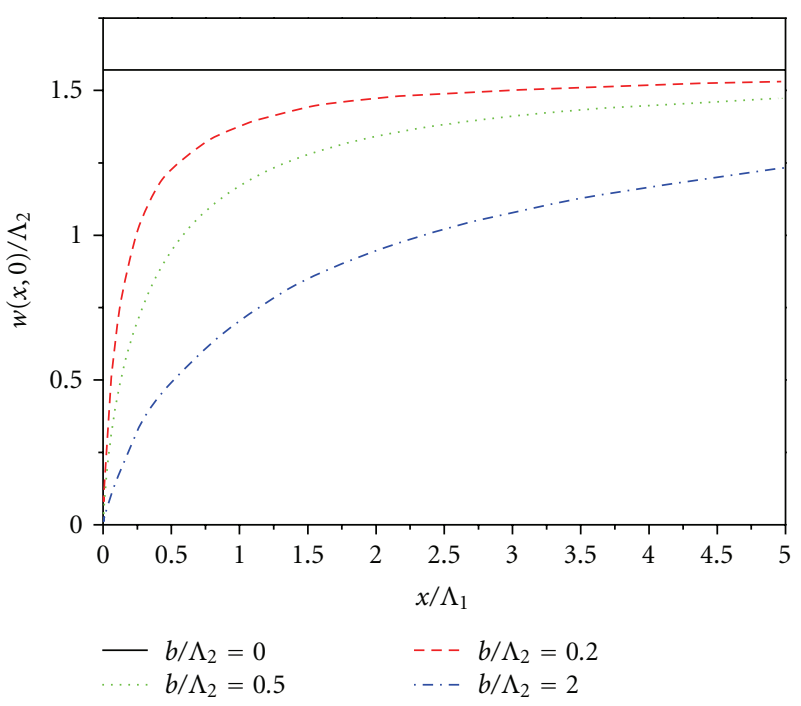

FIgURE 8: Surface displacement under a concentrated shear load.

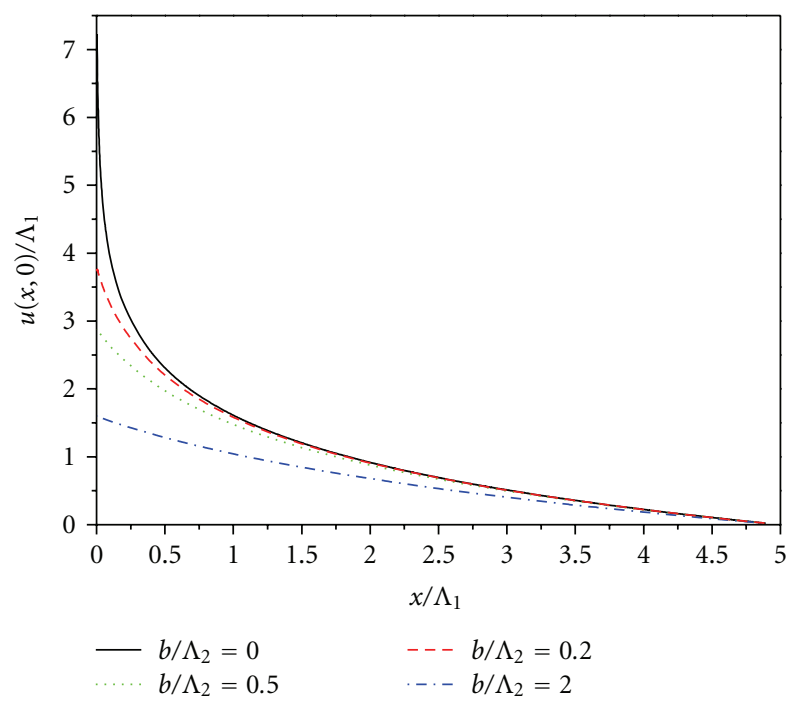

FIGURE 9: Surface indentation under a concentrated shear load.

account. The indent depth decreases as the surface stress increases.

Though only two special cases have been considered in contact problems with surface stresses, based on the solution of a point force applied on a semi-infinite body, one can deal with various types of contact problems by solving an integral equation, as those have been done in the classical contact mechanics.

\section{Conclusions}

The two-dimensional nanocontact problems for elastic bulk materials subjected to surface shear loads are investigated with completed surface stress effect (nonzero surface tension and surface elastic properties). A set of analytical solutions are presented by using Fourier integral transform method. 
The closed-form solution can be derived for the case of a half-plane. For the two particular cases of a uniform distributed shear load and a concentrated shear force, the results are analyzed in detail and compared with the classical linear elastic solutions. It is found that the surface elasticity theory illuminates some interesting characteristics of nanocontact problems, which are distinctly different from the classical solutions of elasticity without surface effects. The contact stresses and the displacements of the deformed surface change smoothly across the loading boundary; moreover the stress and the indent depth show a significant dependence on the surface stress. Therefore the effects of surface stresses should be considered for nanocontact problems.

\section{Acknowledgments}

The supports from the National Natural Science Foundation (Grant no. 11062004), the Natural Science Foundation of Gansu province (1010RJZA021) and the Doctoral Foundation of Lanzhou University of Technology are acknowledged.

\section{References}

[1] K. L. Johnson, Contact Mechanics, Cambridge University Press, Cambridge, Mass, USA, 1985.

[2] G. F. Wang and X. Q. Feng, "Effects of surface stresses on contact problems at nanoscale," Journal of Applied Physics, vol. 101, Article ID 013510, 2007.

[3] M. E. Kassner, S. Nemat-Nasser, Z. Suo et al., "New directions in mechanics," Mechanics of Materials, vol. 37, no. 2-3, pp. 231-259, 2005.

[4] Y. Huang, F. Zhang, K. C. Hwang, W. D. Nix, G. M. Pharr, and G. Feng, "A model of size effects in nano-indentation," Journal of the Mechanics and Physics of Solids, vol. 54, no. 8, pp. 1668 1686, 2006.

[5] R. Dingreville, J. Qu, and M. Cherkaoui, "Surface free energy and its effect on the elastic behavior of nano-sized particles, wires and films," Journal of the Mechanics and Physics of Solids, vol. 53, no. 8, pp. 1827-1854, 2005.

[6] A. J. Kulkarni and M. Zhou, "Surface-effects-dominated thermal and mechanical responses of zinc oxide nanobelts," Acta Mechanica Sinica, vol. 22, pp. 217-224, 2006.

[7] B. Movchan and I. S. Jones, "Asymptotic and numerical study of a surface breaking crack subject to a transient thermal loading," Acta Mechanica Sinica, vol. 22, pp. 22-27, 2006.

[8] G. F. Wang and X. Q. Feng, "Interface effects on effective elastic moduli of nanocrystalline materials," Materials Science and Engineering A, vol. 363, pp. 1-8, 2003.

[9] Z. Y. Ou, G. F. Wang, and T. J. Wang, "Effect of residual surface tension on the stress concentration around a nanosized spheroidal cavity," International Journal of Engineering Science, vol. 46, pp. 475-485, 2008.

[10] Z. Y. Ou, G. F. Wang, and T. J. Wang, "Elastic fields around a nanosized spheroidal cavity under arbitrary uniform remote loadings," European Journal of Mechanics A/Solids, vol. 28, pp. 110-120, 2009.

[11] Z. Y. Ou, G. F. Wang, and T. J. Wang, "An analytical solution for the elastic fields near spheroidal nano-inclusions," Acta Mechanica Sinica, vol. 25, pp. 821-830, 2009.

[12] Z. Y. Ou and S. D. Pang, "A screw dislocation interacting with a coated nano-inhomogeneity incorporating interface stress,"
Materials Science and Engineering A, vol. 528, no. 6, pp. 27622775, 2011.

[13] M. E. Gurtin and A. Ian Murdoch, "A continuum theory of elastic material surfaces," Archive for Rational Mechanics and Analysis, vol. 57, no. 4, pp. 291-323, 1975.

[14] M. E. Gurtin and A. Ian Murdoch, "Surface stress in solids," International Journal of Solids and Structures, vol. 14, no. 6, pp. 431-440, 1978.

[15] R. E. Miller and V. B. Shenoy, "Size-dependent elastic properties of nanosized structural elements," Nanotechnology, vol. 11, no. 3, pp. 139-147, 2000.

[16] R. C. Cammarata, K. Sieradzki, and F. Spaepen, "Simple model for interface stresses with application to misfit dislocation generation in epitaxial thin films," Journal of Applied Physics, vol. 87, no. 3, pp. 1227-1234, 2000.

[17] V. B. Shenoy, "Atomistic calculations of elastic properties of metallic fcc crystal surfaces," Physical Review B, vol. 71, Article ID 094104, 2005.

[18] W. Gao, S. Yu, and G. Huang, "Finite element characterization of the size-dependent mechanical behaviour in nanosystems," Nanotechnology, vol. 17, no. 4, pp. 1118-1122, 2006.

[19] W. X. Zhang and T. J. Wang, "Effect of surface energy on the yield strength of nanoporous materials," Applied Physics Letters, vol. 90, Article ID 063104, 2007.

[20] W. X. Zhang, T. J. Wang, and X. Chen, "Effect of surface stress on the asymmetric yield strength of nanowires," Journal of Applied Physics, vol. 103, Article ID 123527, 2008.

[21] X. J. Zhao and R. K. N. D. Rajapakse, "Analytical solutions for a surface-loaded isotropic elastic layer with surface energy effects," International Journal of Engineering Science, vol. 47, pp. 1433-1444, 2009.

[22] F. Q. Yang and, "Size-dependent effective modulus of elastic composite materials: spherical nanocavities at dilute concentrations," Journal of Applied Physics, vol. 95, pp. 3516-3520, 2004. 

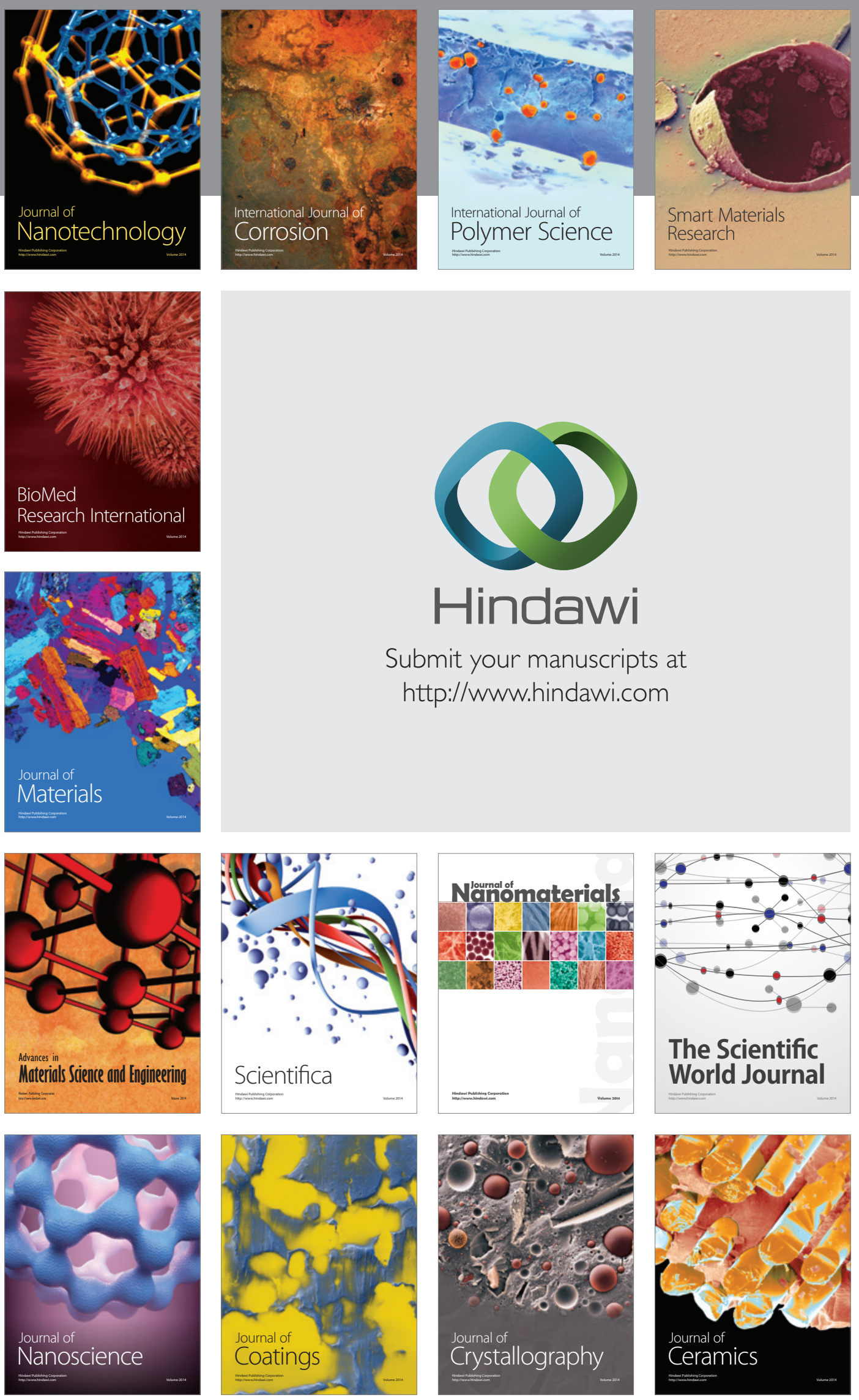

The Scientific World Journal

Submit your manuscripts at

http://www.hindawi.com

\section{World Journal}

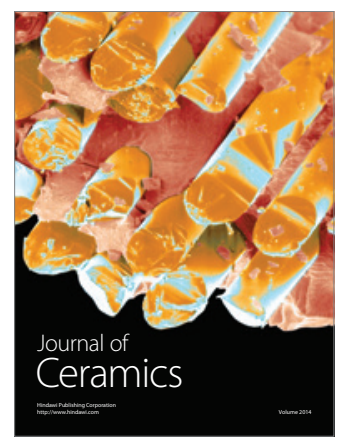

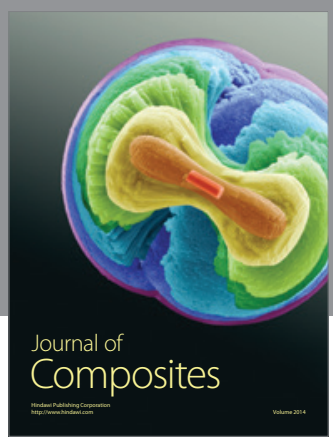
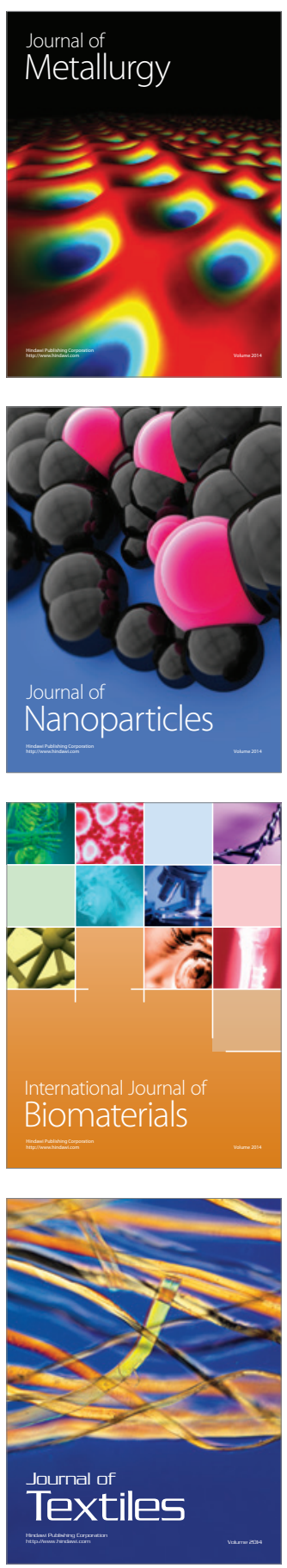